\title{
St. Gallen/Vienna 2017: A Brief Summary of the Consensus Discussion about Escalation and De-Escalation of Primary Breast Cancer Treatment
}

\author{
Michael Gnant $^{\mathrm{a}} \quad$ Nadia Harbeck $^{\mathrm{b}} \quad$ Christoph Thomssen $^{\mathrm{c}}$ \\ ${ }^{a}$ Department of Surgery and Comprehensive Cancer Center Vienna, Medical University of Vienna, Vienna, Austria; \\ ${ }^{b}$ Breast Center, Department of Obstetrics and Gynecology, University of Munich (LMU), Munich, Germany; \\ ${ }^{c}$ Department of Gynecology, Martin-Luther-University, Halle/Saale, Germany
}

\section{Keywords}

Early breast cancer - Neoadjuvant systemic therapy .

Adjuvant treatment - Endocrine therapy .

Chemotherapy · Bisphosphonates - Denosumab ·

Breast surgery · Sentinel node - Axillary dissection .

Radiotherapy - Targeted therapy

\section{Summary}

For the second time, the St. Gallen Consensus Conference on early breast cancer treatment standards took place in Vienna, Austria, where it will remain for the foreseeable future (next date: March 20-23, 2019). With the probably most prominent line-up of global breast cancer experts and more than 3,000 participants from over 100 countries, the 2017 St. Gallen/Vienna conference again was a huge success. A generation change took place with respect to the Conference Co-Chairpersons. Traditionally, the experts from all continents reviewed publications from the past 2 years, and discussed whether new diagnostic or therapeutic means were ready for routine everyday practice. This year, the conference's main theme was 'Escalating and Deescalating Treatment', and the traditional panel votings clarified a number of issues in this respect. Several subjects of all breast cancer modalities were further de-escalated (surgery: 'no ink on tumor' clearly confirmed as standard; resection within new limits after neoadjuvant systemic therapy; axillary dissection may also be avoided after mastectomy under certain circumstances; radiotherapy: hypofractionation is standard of care in breast conserving therapy; chemotherapy: can be avoided in low-risk patients). However, others were escalated: surgery: after neoadjuvant treat- ment and after mastectomy a positive sentinel node leads to axillary dissection; radiotherapy: regional nodes have to be irradiated in 4+ nodes situations; adjuvant therapy: bisphosphonates as standard for postmenopausal women. There was no clear panel opinion on the optimal use of multigenomic assays. As always, the panel recommendations are strictly opinion-based, and try to depict the 'usual' treatment for the 'average' patients. This rapid report by the editors-in-chief of BREAST CARE summarizes the results of the 2017 international panel votings with respect to loco-regional systemic treatment, and does not intend to replace the official St. Gallen Consensus publication.

(c) 2017 S. Karger GmbH, Freiburg

\section{Introduction}

The St. Gallen Consensus Conference 2017 (March 15-18) was again held in Vienna, Austria. Panel discussion and voting were chaired by Giuseppe Curigliano and Eric Winer who were supported by 8 co-chairs. Voting focused again on therapy recommendations for early breast cancer which are based on evidence as well as clinical expertise of the international faculty from 23 countries from all 5 continents (table 1). The panel openly disclosed any potential conflict of interest (www.oncoconferences.ch); the COI committee was once more chaired by Harold Burstein (Boston, MA, USA). It was recognized that individual panel members may have financial relationships with commercial entities engaged in re-

\section{KARGER}

() 2017 S. Karger GmbH, Freiburg 
Table 1. Members of the St. Gallen 2017 international breast cancer consensus panel

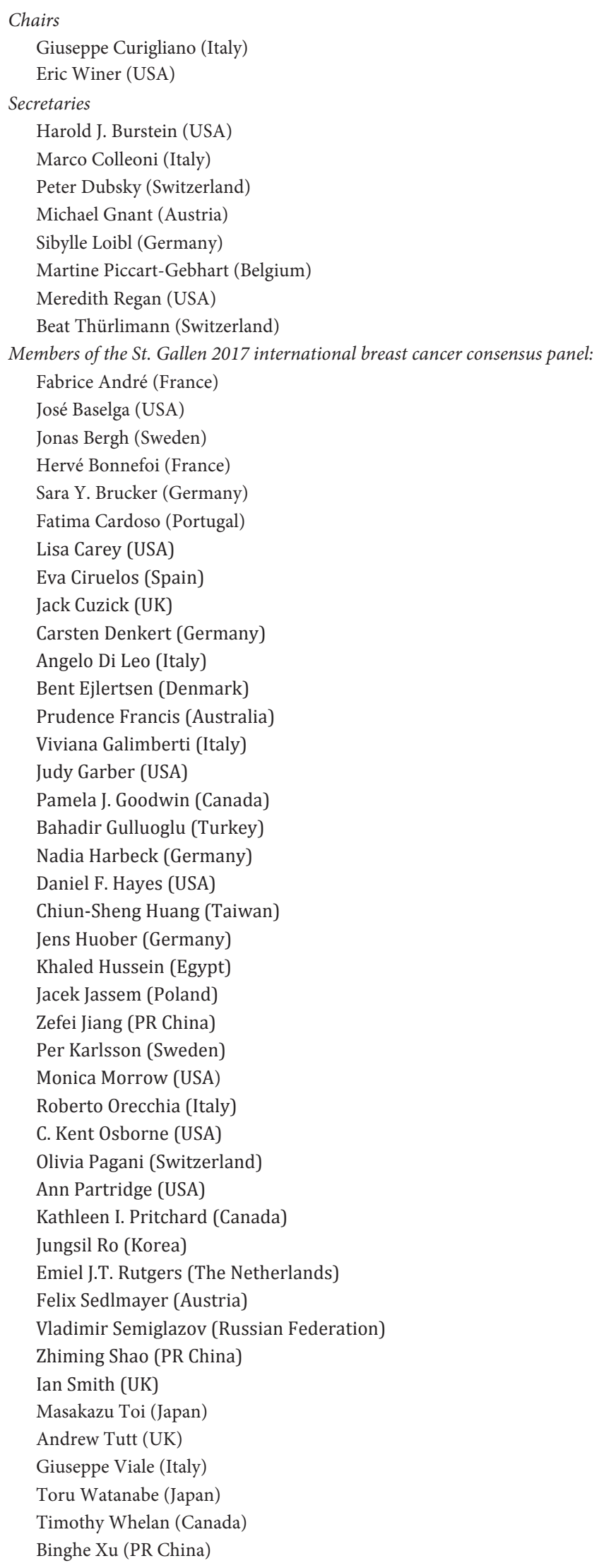

search, innovation, and education. None of the declared conflicts were judged to substantially impact the voting procedure or warrant exclusion of a panel member. However, members with a specific COI were asked to abstain from voting at certain questions.

The motto of this year's panel discussions and voting ('Consensus and Controversy') was: 'Escalating and De-Escalating Treatment in Early Breast Cancer across Subtypes and Treatment Modalities'. About 3,000 participants from 105 countries saw 3 days of high-level educational lectures addressing the local and systemic therapy as well as imaging issues. However, half of the sessions dealt with biology, pathology, and translational research issues. Interestingly, and against contrary trends of other disease-specific conferences, again, a large number of participants from overseas, particularly China, Japan, and the USA, attended and made the meeting the probably most important international breast cancer meeting on European soil from a global perspective.

The Saturday morning consensus panel was again co-chaired by Giuseppe Curigliano (Italy) and Eric Winer (USA), who also moderated the discussion and the voting. More than 200 questions (of which 145 were finally selected for the voting) had been developed and exchanged by the panelists beforehand, which reduced the public debate and allowed to spend more time on the panel discussion with regard to the controversial and important subjects for breast cancer care around the world. It was agreed that clinical trials provide the evidence for general recommendations in clinical decision-making; however, it was also stated that evidence from randomized clinical trials does not cover all controversies that arise in treating individuals. Thus, expert opinion had to be used when data were lacking. This is the unique feature of the St. Gallen International Consensus.

In general, the panelists were asked to cast their vote using 3 possible answers: yes / no / abstain. However, due to the complexity of some questions, more options were given in certain instances. 'Abstain' was to be used in case of insufficient data, no personal expertise on the particular issue, or a conflict of interest of a given panelist. After each vote, the answers were summarized in percentages. This report summarizes the original voting questions and resulting percentages of the St. Gallen/Vienna panel discussion on Saturday March 23, 2017 (abstaining votes are not always described in this report).

\section{Surgery of the Primary Tumor}

Again, locoregional treatment aspects were a major topic of this year's St. Gallen/Vienna Consensus: Following extensive discussion, in general it can be noted that there was some further de-escalating in surgical aspects of tumor resection, and some escalation of loco-regional radiotherapy. Again, the margin issue was undisputedly clarified as 'no ink on tumor' for primary surgery of invasive tumors, but this time a majority voted for $2 \mathrm{~mm}$ margins in DCIS (62\%), whereas $35 \%$ found 'no ink on DCIS' sufficient ( $4 \%$ abstained). The panel again was very clear (94\%) that margins should not depend on tumor biology. 
Table 2. Questions and answers regarding clinical utility of multigene tests

\begin{tabular}{|c|c|c|c|}
\hline Test & $\begin{array}{l}\text { ER+ HER2- pN0 } \\
\text { prognosis year 1-5 } \\
\text { yes / no / abstain }\end{array}$ & $\begin{array}{l}\text { ER+ HER2- pN+ } \\
\text { prognosis year 1-5 } \\
\text { yes / no / abstain }\end{array}$ & $\begin{array}{l}\text { ER+ HER2- pN+ } \\
\text { chemotherapy indication } \\
\text { yes / no / abstain }\end{array}$ \\
\hline Breast Cancer Index $(\mathrm{BCI})^{\circledR}$ & $60 \% / 20 \% / 20 \%$ & $43.3 \% / 33.3 \% / 23.3 \%$ & $8.1 \% / 64.9 \% / 27 \%$ \\
\hline EndoPredict ${ }^{\circledR}\left(\right.$ EPclin $\left.^{\circledR}\right)$ & $70 \% / 20 \% / 10 \%$ & $55.6 \% / 16.7 \% / 27.8 \%$ & $15.8 \% / 52.6 \% / 31.6 \%$ \\
\hline MammaPrint ${ }^{\circledR}$ & $81.3 \%$ / $6.3 \% / 12.5 \%$ & $42.9 \%$ / 35.7\% / 21.4\% & $40 \% / 50 \% 10 \%$ \\
\hline Onkotype DX ${ }^{\circledR}$ & $93.8 \% / 6.3 \% / 0 \%$ & $60 \% / 30 \% / 10 \%$ & $58.6 \% / 31 \% / 10.3 \%$ \\
\hline Prosigna ${ }^{\circledR}$ & $80 \% / 0 \% / 20 \%$ & $75 \% / 12.5 \% / 12.5 \%$ & $46.7 \%$ / $53.3 \% / 0 \%$ \\
\hline
\end{tabular}

Once more, breast conserving surgery was confirmed as intended standard of care of surgery for breast cancer, this time almost unequivocally in cases of multifocal ( $97 \%$ yes, $3 \%$ no) or multicentric (61\% yes, 33\% no, $6 \%$ abstain) disease, provided that clear margins can be reached and adequate radiotherapy is planned.

Finally, after decades of discussion, the panel this time also deescalated surgery after neoadjuvant systemic treatment: $82 \%$ of panelists voted that surgical resection should be oriented on the post-neoadjuvant extent of the tumor, and only $14 \%$ of panelists insisted that the original (pre-treatment) tumor bed needs to be resected ( $4 \%$ abstained).

After neoadjuvant systemic therapy without multifocal residual disease in the pathology specimen, $96 \%$ of the panel felt that 'no ink on tumor or DCIS' would be sufficient ( $2 \mathrm{~mm}$ clearance: $4 \%$ ). The panel was a bit more cautious, albeit still rather clear, in situations of multifocal residual disease after neoadjuvant systemic therapy: 55\% of the panel felt that 'no ink on tumor or DCIS' would be appropriate, whereas $28 \%$ felt more comfortable with a $2 \mathrm{~mm}$ margin (2-5 mm margin: 7\%, >5 mm margin: 3\%, 7\% abstain). Nipplesparing was considered a safe procedure after neoadjuvant systemic therapy ( $80 \%$ yes, $4 \%$ no).

\section{Pathology and Multigene Tests}

The consensus questions regarding pathology and multigene testing were summarized under the heading ' when is traditional pathology (stage, grade, LVI, ER/PR/HER2) not informative enough?' $100 \%$ of the panelists agreed that the distinction between luminal A-like and luminal B-like by immunohistochemistry describes important categories in the biology of luminal breast cancer. $80 \%$ agreed that these two categories should be used for therapy decisions and $67 \%$ agreed that IHC can be used to approximate multigene testing in this context. While $79 \%$ agreed that ER, PR, and 'high' Ki67 can be used to distinguish between luminal A-like and luminal B-like breast cancer, a majority of $64 \%$ stated that subtype can be more appropriately determined by a multigene test. Only $29 \%$ of the panelists believed that the evaluation of tumorinfiltrating lymphocytes (TILs) should be reported in triple-negative and HER2+ early breast cancer (EBC).

The questions regarding multigene tests specifically addressed 5 tests, i.e. Breast Cancer Index ${ }^{\circledR}$, EndoPredict ${ }^{\circledR}\left(\right.$ EPclin $\left.^{\circledR}\right)$, MammaPrint ${ }^{\circledR}$, Oncotype DX ${ }^{\circledR}$, and Prosigna ${ }^{\circledR}$. The majority of panelists (86\%) did not consider multigene testing necessary in pT1a-b
pN0 ER+ PR+ HER2- low Ki67 and low grade EBC. Overall, outside of this low-risk subgroup, panelists agreed that all multigene tests provide valuable information on prognosis and risk, thus helping to omit chemotherapy in ER+ HER2- pN0 EBC. In pN+ disease (i.e. in 1-3 involved lymph nodes), agreement regarding prognosis was lower and some tests even received a 'no' vote for using it in order decide about chemotherapy in this population (table 2). Only $46 \%$ of the panelists (no 50\%) believed that multigene signatures provide valuable information for decision regarding extended endocrine therapy.

\section{Adjuvant Endocrine Therapy in Premenopausal Patients}

Similarly to the last St. Gallen Consensus in 2015 [1], and still affected by the data on ovarian function suppression as adjuvant treatment option in premenopausal patients, a substantial part of the discussion was again focused on this topic $[2,3]$. The panel discussed several clinical situations as indication by itself for ovarian function suppression (OFS), and there was a strong agreement that involvement of 4 or more axillary lymph nodes alone would argue in favor of OFS (yes $84 \%$, no $12 \%$ ); a majority considered also age $<35$ years alone as a reasonable indication for OFS (yes $77 \%$, no $19 \%$ ); however, with regard to premenopausal estrogen levels there was less agreement (yes $60 \%$, no $34 \%$ ). In summary, the panel agreed widely that some, but not all patients should receive OFS in combination with aromatase inhibitors (AI) (yes $92 \%$, no $6 \%)$.

\section{Adjuvant Endocrine Therapy in Postmenopausal Patients}

After years of agreement that AI should be incorporated at some point into endocrine adjuvant treatment, the panel of 2017 tended to de-escalate this strong recommendation. Only a weak majority pleaded for inclusion of an $\mathrm{AI}$ at some point in all postmenopausal patients (yes 51\%, no 44\%). More panelists considered HER2 positivity as an argument for an AI (yes 62\%, no 34\%).

Although upfront AI therapy probably is already reality in many countries, the panel discussed and voted whether AI therapy should be started upfront; there was a high agreement in favor of upfront therapy for patients at higher risk (yes $94 \%$, no 
Table 3. Multigene tests and their impact on chemotherapy indications

\begin{tabular}{lll}
\hline Test & $\begin{array}{l}\text { Omission of chemotherapy in N0 } \\
\text { yes / no / abstain }\end{array}$ & $\begin{array}{l}\text { Omission of chemotherapy in N+ (1-3 LN) } \\
\text { yes / no / abstain }\end{array}$ \\
\hline EndoPredict $^{\circledR}\left(\right.$ EPclin $\left.^{\circledR}\right)$ low risk & not voted & $20 \% / 66 \% / 14 \%$ \\
MammaPrint $^{\circledR}$ low risk & not voted & $55.1 \% / 34.7 \% / 10.2 \%$ \\
Onkotype DX ${ }^{\circledR}$ low risk & $87.8 \% / 10.2 \% / 2 \%$ & $55.6 \% / 33.3 \% / 11.1 \%$ \\
Onkotype DX ${ }^{\circledR}$ intermediate risk & $22.4 \% / 67.3 \% / 10.2 \%$ & $6.3 \% / 87.5 \% / 6.3 \%$ \\
Prosigna ${ }^{\circledR}$ low risk & not voted & $30.8 \% / 50 \% / 19.2 \%$ \\
\hline
\end{tabular}

$6 \%$ ) and for patients with lobular cancer (yes 78\%, no 14\%), but not in any patient (yes $56 \%$, no $40 \%$ ). A switch from AI to tamoxifen after 2 years should not be performed in all patients (yes 19\%, no $72 \%)$.

\section{Duration of Adjuvant Endocrine Therapy}

It is well known that particularly in ER+ breast cancer patients half of all recurrences occur more than 5 years from primary diagnosis. Although modern treatments might be highly effective, in the Oxford meta-analysis this accumulates to $25 \%$ risk of recurrences after 10 years in patients with ER+ breast cancer [4]. Therefore, extending adjuvant therapy beyond the 5 th year was always discussed and it was studied in several clinical trials; it is well accepted for high-risk patients. Duration of endocrine therapy is an issue of substantial therapy escalation; thus, well-founded indication is of high importance. So far, a survival benefit was shown only for patients with node-positive disease [5].

Although multigene signatures may well predict late recurrences that occur 5 years from primary diagnosis and beyond [68], only a minority argued in favor of triggering extended adjuvant treatment by the results of such signatures (yes 46\%, no 50\%).

Provided an indication exists for therapy beyond the first 5 years, there was high agreement that patients at moderate or high risk of recurrence after 5 years of adjuvant therapy involving a switch from tamoxifen to an AI should be recommended to continue AI to a cumulative total of 5 years AI (yes 89\%, no 6\%); some panelists voted even for prolonging AI by further 5 years (yes $66 \%$, no $23 \%$ ). Tamoxifen was not considered to be helpful in this particular situation; only $8 \%$ of the panelists would decline any further endocrine therapy.

In daily practice, we treat many patients who had received upfront AI adjuvant therapy for 5 years. Based on recent results, two thirds of the panelists thought that after 5 years of initial AI it is not necessary to provide further endocrine therapy. However, total agreement among the panelists was only achieved as far as that the duration of AI should depend upon tolerance and absolute risk (yes $98 \%$, no $2 \%$ ). A switch to tamoxifen was favored only by a minority (yes 26\%, no 59\%), the majority would recommend continuation of AI of 3-5 years after initial tamoxifen (yes 65\%, no $28 \%$ ).

For patients who remain premenopausal during the first 5 years, prolongation of tamoxifen to 10 years should be restricted to patients at high risk at presentation (yes $86 \%$, no $10 \%$ ). Again axillary lymph node involvement may be predictive for survival benefit [9].

\section{Adjuvant and Neoadjuvant Chemotherapy}

The consensus questions regarding chemotherapy in EBC were summarized under the heading 'which women should receive adjuvant chemotherapy?' $96.1 \%$ of the panelists agreed that treatment decisions about both prognosis and potential benefits of chemotherapy in $\mathrm{N} 0$ disease can be aided by tumor biology as defined by IHC as well as by a multigene risk predictor. As relative indications for adjuvant chemotherapy, agreement was seen for the following factors: histological grade 3 (yes 90.6\%), any positive node (yes $68.5 \%$, no $31.5 \%$ ), high Ki67 (yes $84.9 \%$ ), age < 35 years (yes $55.6 \%$, no $44.2 \%$ ), extensive lympho-vascular invasion (yes $67.9 \%$, no $30.2 \%$ ), and low hormone receptor staining (yes 91.1\%). 57.1\% (no $34.7 \%$ ) believed that chemotherapy should be recommended in all $\mathrm{N} 0$ and $\mathrm{N}+$ patients with poor prognosis biology.

In patients with luminal B-like tumors, multigene tests were voted separately regarding their support of the decision to omit chemotherapy (table 3).

Next adjuvant chemotherapy in luminal B patients was discussed. Here, $52.1 \%$ of the panelists supported the use of anthracyclines and taxanes, whereas $39.6 \%$ did not. Only $23.1 \%$ thought that chemotherapy should comprise 6 cycles of the same therapy such as $6 \times \mathrm{EC}, \mathrm{AC}$ or TC whereas $63.5 \%$ disagreed.

For triple-negative breast cancer (TNBC), only 55.8\% agreed (no $40.4 \%$ ) with regimens containing anthracyclines and taxanes for stage I disease, whereas $94 \%$ agreed for these two substances being standard in stage II and III TNBC. $86.3 \%$ did not accept the use of a platinum-based regimen for adjuvant chemotherapy in all patients with TNBC. For patients with germline mutations, $47.1 \%$ would consider a platinum-based regimen whereas $43.1 \%$ would not. $78 \%$ of the panelists agreed (no 20\%) that chemotherapy can be avoided in pT1a pN0 TNBC. Only 37.7\% thought that dosedense regimens should be preferred in TNBC (no 54.9\%).

For HER2+ EBC with positive lymph nodes, $86.2 \%$ agreed that chemotherapy should always be given. $62 \%$ wanted an anthracycline and $87.8 \%$ a taxane to be part of the chemotherapy backbone. In HER2+ node-negative EBC, in pT1a disease only 33.3\% (no $62.5 \%$ ) saw an indication for anti-HER2 therapy, whereas $87.5 \%$ in pT1b disease and $94.1 \%$ in pT1c supported the use of anti-HER2 therapy. In HER2+ disease (according to ASCO/CAP guidelines), $92 \%$ considered the combination of paclitaxel and trastuzumab as a reasonable adjuvant option. For N0 disease, agreement depended on size of the primary tumor with $88.5 \%$ agreement for tumors $<1$ $\mathrm{cm}, 78.8 \%$ for tumors $1-2 \mathrm{~cm}$, and only $50 \%$ for tumors $2-3 \mathrm{~cm}$ (no $44 \%$ ). 4 cycles of the combination of docetaxel and cyclophos- 
phamide together with trastuzumab were also accepted as a reasonable option for node-negative HER2+ disease. There was $53.8 \%$ support for the use of trastuzumab biosimilars for (neo-)adjuvant anti-HER2 therapy - provided there was an approval - whereas $17.3 \%$ disagreed and $28.8 \%$ abstained.

Supporting a paradigm change in EBC, there was $94.1 \%$ approval for neoadjuvant therapy being the preferred option in stage II-III HER2+ disease and $92.5 \%$ approval for neoadjuvant therapy being the preferred option in stage II-III disease TNBC.

For stage II-III HER2+ disease, only 34.8\% (no 56.5\%) accepted a taxane and trastuzumab alone as a neoadjuvant therapy option whereas $84.3 \%$ accepted a taxane together with trastzuzumab and pertuzumab. In patients who received neoadjuvant chemotherapy with dual HER2 blockade (trastuzumab and pertuzumab), there was $88 \%$ support for completing anti-HER2 therapy with adjuvant trastuzumab alone for 1 year and only $6.1 \%$ thought that adjuvant therapy should also consist of trastuzumab and pertuzumab (no $69.4 \%)$

For stage II TNBC, $70.8 \%$ considered a regimen containing platinum or alkylating agents as the preferred neoadjuvant regimen irrespective of BRCA status. There was $74.5 \%$ agreement that the preferred regimen should include an anthracycline followed by a taxane in a non-dose dense fashion. $67.3 \%$ did not believe that the preferred regimen should include nab-paclitaxel followed by EC, but $56.3 \%$ believed that nab-paclitaxel followed by EC was a reasonable neoadjuvant regimen in TNBC irrespective of BRCA status.

One additional topic was the question 'which women should receive additional therapy after neoadjuvant treatment?' Given the case of residual disease $>1 \mathrm{~cm}$ and/or a positive node at surgery following neo-adjuvant (anthracycline-, taxane-, and alkylatorbased) chemotherapy for TNBC, $31.1 \%$ opted for no further therapy while $48.9 \%$ proposed capecitabine, $6.7 \%$ platinum, $8.9 \%$ platinum in case of BRCA+, and $4.4 \%$ metronomic chemotherapy. If a clinical trial was available, $90.2 \%$ agreed that this should be proposed to such patients.

\section{Special Populations}

In absence of significant co-morbidity, $94.1 \%$ agreed that there is no absolute age limit for use of chemotherapy but the indication rather depended on disease properties. Scalp cooling was accepted by $83 \%$ of the panelists as an option to prevent hair loss during (neo-)adjuvant chemotherapy.

Genetic testing for high-risk mutations should be considered, after counselling, in patients with a strong family history (yes $96.2 \%$ ), patients $<40$ years at breast cancer diagnosis (yes 75\%), patients $<60$ years with TNBC only (yes $68.6 \%$ ), but not necessarily in all patients $<50$ years at breast cancer diagnosis (yes $18.9 \%$, no 77.4\%). Panelists believed that BRCA 1 or 2 mutations may impact decisions on breast surgery (yes $88.5 \%$ ), on systemic therapies (yes $73.1 \%$, no $23.1 \%$ ), as well as other prophylactic interventions (yes $94.1 \%$ ).

\section{Adjuvant Bone-Targeted Therapies}

Following a recent update of the respective joint ASCO/Cancer Care Ontario Clinical Practice Guideline [10], the panel clearly defined adjuvant bone-targeted agents such as zoledronic acid q 6 months or oral clodronate during adjuvant endocrine therapy as standard of care for postmenopausal patients, aiming at the improvement of disease free survival (DFS), irrespective of bone mineral density ( $76 \%$ yes, $18 \%$ no, $6 \%$ abstain) [11]. The panel was less clear on the same questions for premenopausal women on ovarian function suppression (plus tamoxifen or AI) (53\% yes, 37\% no, 10\% abstain), and voted against adjuvant bisphosphonates for young patients without LHRH agonist therapy (90\% yes, $2 \%$ no, $8 \%$ abstain).

Despite promising results of the ABCSG-18 trial [12], the panel did not endorse that adjuvant denosumab should substitute for bisphosphonates in that treatment situation (30\% yes, $44 \%$ no, $26 \%$ abstain).

\section{Special Issues and Patient Populations}

For elderly patients, the panel was clearer than ever that numerical age is not important for treatment decisions ( $94 \%$ yes). Rather, individual disease factors, patient co-morbidities, overall life expectancy, and patient preference should be important in the decision-making process ( $87 \%$ yes). With respect to radiotherapy after breast conserving surgery, $53 \%$ of the panel felt that radiotherapy may be omitted when multiple co-morbidities are present. $12 \%$ voted that this may be done over the age of $65,27 \%$ over the age of 70 years ( 75 years: $2 \%, 80$ years: $4 \%, 2 \%$ abstain)

The panel was split on the question whether pregnancy could be attempted during the first 5 years following surgery (yes: $38 \%$, no $52 \%$, abstain $10 \%$ )

With respect to male breast cancer, the panel declined the use of AI instead of tamoxifen (yes 16\%, no 69\%, abstain 16\%), but indicated that AI + LHRHa could be an option (yes 55\%, no 23\%, abstain 21\%).

\section{Genetic Testing}

The panel escalated the indications for genetic testing by stating that such testing for high-risk mutations should - after appropriate counselling - be considered for patients with a strong family history (yes $96 \%$, no $2 \%$, abstain $2 \%$ ), for all patients with breast cancer under the age of 40 years (75\%, no $21 \%$, abstain $4 \%$ ).

The panel clearly declined genetic testing for all patients with a breast cancer diagnosis under the age of 50 (yes 19\%, no 77\%), but endorsed genetic testing for high-risk mutations for triple-negative patients under the age of 60 (yes 69\%, 29\% no).

The panel also stated that the presence of BRCA $1 / 2$ mutations may impact decisions of breast surgery (yes $88 \%$, no $8 \%$, abstain $4 \%$ ), systemic therapy (yes $73 \%$, no $23 \%$ ), and other prophylactic interventions (yes $94 \%$, no $4 \%$ ) 


\section{Lifestyle}

The last topic discussed on Saturday morning in Vienna was summarized under the heading 'should breast cancer patients receive specific diet and lifestyle interventions beyond 'ordinary' advice on maintaining healthy lifestyles?' Panelists were asked whether - independent of any lifestyle recommendations that may be offered for rehabilitation, symptom control and/or general health, they would issue additional recommendations with the goal of reducing risk of recurrence and/or death from breast cancer. About two thirds supported dietary advice in keeping with national guidelines (yes 56\%, no 34\%), physical activity (at least 150 min per week) (yes 66\%, no $31 \%$ ), as well as weight loss to a normal BMI (i.e. $20-25 \mathrm{~kg} / \mathrm{m}^{2}$ ) and avoidance of weight gain (providing BMI at least 20) (yes 67\%, no 27\%)

\section{Conclusion}

In summary, St. Gallen/Vienna 2017 was again a highly successful global breast cancer conference. International attendance increased as compared to the previous meeting, to 3,000 participants from 105 countries, and the panel discussions and voting fulfilled the expectations of escalating and de-escalating a number of important issues for the daily care of primary breast cancer patients.
Apparently, the incipient generation change in co-chairs and panelists did not decrease the quality and intensity of the excellent preparatory work of the faculty. In general, the discussions were highlevel and only controversial at certain issues. The process is a role model for field moves towards personalization of breast cancer oncology, and while the St. Gallen/Vienna Consensus process is per definition not a guideline development process because of its opinion-based nature, the collective wisdom of many of the most experienced and distinguished leaders in their respective sub-specialties and research fields will certainly define important guidance and an important source of advice in individual decision-making for many physicians and patients worldwide. We look forward to the final scientific manuscript, as well as to the next St. Gallen Consensus Meeting in Vienna, March 20-23, 2019.

\section{Disclosure Statement}

Michael Gnant reports having received institutional grants during the last 2 years from: AstraZeneca, Novartis, Roche, and Pfizer; and personal honoraria and travel support from: Accelsiors, Roche, Novartis, AstraZeneca, Celgene, Pfizer, GSK, Amgen, OBI-Pharma, Ipsen, and Eisai. Nadia Harbeck reports honoraria or consultation fees from the following entities: Agendia, Amgen, Celgene, Genomic Health, Lilly, MSD, Nanostring, Novartis, Pfizer, Roche, Sandoz. Christoph Thomssen reports receiving honoraria for lectures and advisory board membership from Amgen, Astra-Zeneca, Celgene, Genomic-Health, Nanostring, Novartis, Pfizer, and Roche.

\section{References}

1 Gnant M, Thomssen C, Harbeck N: St. Gallen/Vienna 2015: A brief summary of the consensus discussion. Breast Care 2015;10:124-130.

2 Francis PA, Regan MM, Fleming GF, et al.: Adjuvant ovarian suppression in premenopausal breast cancer. N Engl J Med 2015;372:436-446.

3 Pagani O, Regan MM, Walley BA, et al.: Adjuvant exemestane with ovarian suppression in premenopausal breast cancer. N Engl J Med 2014;371:107-118.

4 Early Breast Cancer Trialists' Collaborative Group (EBCTCG), Davies C, Godwin J, et al.: Relevance of breast cancer hormone receptors and other factors to the efficacy of adjuvant tamoxifen: patient-level metaanalysis of randomised trials. Lancet 2011;378:771-784.

5 Goss PE, Ingle JN, Martino S, et al.: Randomized trial of letrozole following tamoxifen as extended adjuvant therapy in receptor-positive breast cancer: updated findings from NCIC CTG MA.17. J Natl Cancer Inst 2005;97:1262-1271.
6 Sgroi DC, Sestak I, Cuzick J, et al.: Prediction of late distant recurrence in patients with oestrogen-receptorpositive breast cancer: a prospective comparison of the breast-cancer index (BCI) assay, 21-gene recurrence score, and IHC4 in the TransATAC study population. Lancet Oncol 2013;14:1067-1076.

7 Dubsky P, Brase JC, Jakesz R, et al.: The EndoPredict score provides prognostic information on late distant metastases in ER+/HER2- breast cancer patients. Br J Cancer 2013;109:2959-2964

8 Filipits M, Nielsen TO, Rudas M, et al.: The PAM50 risk-of-recurrence score predicts risk for late distant recurrence after endocrine therapy in postmenopausal women with endocrine-responsive early breast cancer. Clin Cancer Res 2014;20:1298-1305.

9 Ribnikar D, Sousa B, Cufer T, Cardoso F: Extended adjuvant endocrine therapy - a standard to all or some? Breast 2017;32:112-118.
10 Dhesy-Thind S, Fletcher GG, Blanchette PS, et al.: Use of adjuvant bisphosphonates and other bone-modifying agents in breast cancer: a Cancer Care Ontario and American Society of Clinical Oncology clinical practice guideline. J Clin Oncol 2017; DOI: 10.1200/ JCO.2016.70.7257.

11 Hadji P, Coleman RE, Wilson C, et al.: Adjuvant bisphosphonates in early breast cancer: consensus guidance for clinical practice from a European panel. Ann Oncol 2016;27:379-390.

12 Gnant M, Pfeiler G, Dubsky PC, et al.: Adjuvant denosumab in breast cancer (ABCSG-18): a multicentre, randomised, double-blind, placebo-controlled trial. Lancet 2015;386:433-443. 